\title{
Capsule Commentary on Volandes et al., Use of Video Decision Aids to Promote Advance Care Planning in Hilo, Hawai'i
}

\author{
Jeffrey L. Jackson, MD MPH \\ Medical College of Wisconsin and Zablocki VAMC, Milwaukee, WI, USA.
}

J Gen Intern Med 31(9):1079

DOI: $10.1007 / \mathrm{s} 11606-016-3785-0$

(c) Society of General Internal Medicine 2016

\begin{abstract}
A dvanced care planning is a shared medical decision tool to assure that care provision is concordant with the patient's wishes. ${ }^{1}$ In this study, Volandes and colleagues collaborated with the Hawaii Service Association to implement video decision aids for care planning ${ }^{2}$ across all islands in the state. In addition, the state included this as a pay-for-quality measure for the state, and providers were eligible for financial incentives for documenting advanced care planning. Not surprisingly, the rate of care planning went up significantly, from $3.2 \%$ to $39.9 \%$. Given that the study cohort was patients aged 75 years or older, the surprise isn't the improvement in care planning, but that this intervention still left over two-thirds of the elderly with no advanced care planning. The authors correctly point out that a study limitation was the lack of tracking to see which providers offered the videos and which patients actually viewed them. While the study had an impressive increase in patients choosing hospice, it is impossible to definitively assess the video's impact on specific patient behavior.

Modern technology allows providers to include an array of platforms to communicate with their patients. Previous studies suggest they might be helpful with minority populations. $^{3,4}$ This intervention was a video decision tool, but one could easily see motivated health systems hosting these videos on their webpages, on their Facebook accounts and even pushing them to patients. As the current, computer savvy generation ages, one can imagine innumerable opportunities for asynchronous communication
\end{abstract}

and education. This study is a good first step towards assessing the potential impact of video decision aids. Future research needs to provide more granular, patientcentered data, which is lacking from this intervention. Given that this is a patient-centered decision aid, it is ironic that the patient's voice is missing from this study. We don't know who watched the video or their reaction to it. Hawaii has a very distinct culture; creating videos that are culturally sensitive and specific will be a challenge for providers wishing to use them with their patients. It would have been very interesting and potentially enlightening to have assessed a kamaàina (native Hawaiian) reaction to a video created by Boston Haole (Hawaii newcomer).

Corresponding Author: Jeffrey L. Jackson, MD MPH; Medical College of Wisconsin and Zablocki VAMC, Milwaukee, WI, USA (e-mail: jeffrey.jackson6@va.gov).

\section{Compliance with Ethical Standards:}

Conflicts of Interest: The author has no conflicts of interest with this article.

\section{REFERENCES}

1. Institute of Medicine. Dying in America, improving quality and honoring individual preferences near the end of life. Washington DC: The National Academies Press; 2014.

2. Volandes AE, Paasche-Orlow MK, Davis AD, Eubanks R, El-Jawahri A, Seitz R. Use of video decision aids to promote advance care planning in Hilo, Hawai'i. J Gen Intern Med. 2016. doi:10.1007/s11606-016-3730-2.

3. Nathan AG, Marshall IM, Cooper JM, Huang ES. Use of decision aids with minority patients: a systematic review. J Gen Intern Med. 2016;31(6):66376. doi:10.1007/s11606-016-3609-2.

4. Sajid S, Kotwal AA, Dale W. Interventions to improve decision making and reduce racial and ethnic disparities in the management of prostate cancer: a systematic review. J Gen Intern Med. 2012;27(8):1068-78. doi:10.1007/ s11606-012-2086-5.

Published online June 22, 2016 